\title{
STRUCTURALISM, EMPIRICISM, AND NEWMAN'S OBJECTION
}

\author{
OTÁVIO BUENO \\ Department of Philosophy, University of Miami USA \\ otaviobueno@mac.com \\ THOMAS MEIER \\ Munich Center for Mathematical Philosophy, Ludwig-Maximilians University GERMANY \\ meier060782@googlemail.com
}

\begin{abstract}
Newman's objection can be used to argue that structuralism fails to specify a unique structure for the unobservable world, and hence, one can argue, it is ultimately a trivial task to determine the structure that the world ultimately has. Provided there are enough objects, any structure can be made compatible with that structure. We formulate a pragmatically enriched version of structuralism that avoids the Newman objection. For this purpose, we return to Carnap's conception of founded relations, and provide a different interpretation of them. According to Carnap, these are real, experienceable, physical relations. We argue that, when we specify a structural description of a given physical system, if we rely on such founded relations-provided they are properly understood-the threat of the Newman objection is avoided. However, pure structuralism has to be given up, and a form of empiricism can then be advanced. Finally, by using founded relations, we offer a framework in terms of which different conceptions (some realist, some empiricist) can be articulated to avoid the Newman problem as well.
\end{abstract}

Keywords: Newman objection $\bullet$ structuralism $\bullet$ structural realism $\bullet$ empiricism $\bullet$ founded relations $\bullet$ semantic conception.

\section{Introduction}

It is well known that, according to the Newman objection, structuralism runs into a triviality difficulty. We propose a way of addressing this difficulty by resisting the setting in which the objection is posed in the first place. After discussing this objection in Section 2, we identify three significant ingredients that should be used to circumvent it. We then argue, in Section 3, that if structuralism is enriched with resources of a pragmatic nature, it is possible to escape the Newman problem. With such pragmatic traits in place, however, one is forced to leave the territory of pure structuralism. We argue that this can be properly achieved by invoking a Carnapian maneuver. It consists in employing what Carnap (1928) called founded relations, but not with the status Carnap himself assigned to them. After describing this type of relations 
and indicating how the Newman problem can be avoided, a pragmatically enriched and less pure version of structuralism is advanced. This proposal consists in identifying relevant physical structures as part of the description of empirical phenomena in a structuralist way. We explore, in Section 4, some important implications of the framework we propose, and argue that, depending on how it is interpreted, a class of possible responses to the Newman objection — some realist, other anti-realist-are advanced. A brief conclusion follows.

This paper revisits an issue that was also examined in Bueno (2015). In that paper, the role of finiteness in a response to Newman's objection was emphasized, while resisting the use of Carnap's founded relations in the way Carnap envisaged them. In the current paper, these relations play a far more prominent role, but they are also understood in ways that go significantly beyond Carnap's initial proposal. This is needed to avoid the concerns that have been raised against Carnap's own account.

\section{Structuralism and the Newman Objection}

Structuralism is a family of views that emphasizes the importance of structure in the understanding of science. The view has traditionally been associated with realist conceptions (Ladyman 1998 and French 2014), but empiricist reformulations have also been articulated (Bueno 1999 and van Fraassen 2008). We start by considering realist formulations, in the form of structural realism.

\subsection{Structural realism}

Despite the considerable sophistication of the debate about structural realism and the number of variations (see Frigg and Votsis 2011, and French 2014), there are still two main versions of the view, namely, the ontic and the epistemic (Ladyman 1998). The epistemic variant of structural realism (henceforth, ESR) can be expressed as a certain knowledge claim: all that can be known of the world (including its unobservable aspects) is structure. One of the motivations for the view is the consideration to the effect that, as scientific theories change, their ontologies vary considerably (consider, for instance, the fate of phlogiston or the ether). But often, or so the argument goes, the overall (mathematical) structure of the relevant theories is preserved, even though knowledge of the objects that compose that structure may be unavailable (the objects may not exist at all).

In light of the epistemic limitations of ESR, an alternative, metaphysically focused, view has bee proposed: ontic structural realism (OSR). According to it, all that exists (particularly at the ontologically most fundamental level) is structure (La- 
dyman 1998 and French 2014). In the most dramatic, eliminativist formulation of the view, there are no individual objects at the fundamental level (or, at least, no commitment to these objects is required). The challenge is then to articulate a suitable conception of structure for this version of structuralism, given the crucial role that is ultimately played by objects in it. In the end, it is unclear that this can be done (see Arenhart and Bueno 2015).

For the purposes of this paper, our focus lies on ESR, which need not be committed to the elimination of objects. This is the form of structuralism that originally faced a particularly pressing difficulty.

\subsection{The Newman objection}

If all one can know about the world is its structure, there is a concern that such knowledge can be obtained too easily. In fact, provided there are enough objects to begin with, virtually any structure could be the structure of the world. This is the source of the Newman objection against structuralism, an objection that arguably poses a significant problem for epistemic structural realism and, more generally, for structuralist epistemologies broadly conceived (see, for instance, Ainsworth 2009).

The objection was originally raised against Russell's structuralism by the mathematician Max Newman in his review of Russell (1927) (for a discussion, see Demopoulos and Friedman 1985). As Newman points out:

Any collection of things can be organized so as to have the structure $M$, provided there are the right number of them. Hence the doctrine that only structure is known involves the doctrine that nothing can be known that is not logically deducible from the mere fact of existence, except ('theoretically') the number of constituting objects (Newman 1928, p.144).

Newman's point can be easily formulated in set theory. Given a set-theoretic structure $M$, there is always another structure $N$, whose domain is $D$, and which has a substructure $N^{\prime}$ isomorphic to $M$. If $M$ is (a representation of) the structure of the world, and if $D$ is a given collection of objects, it is guaranteed, by set theory, that there will be a structure $N^{\prime}$ isomorphic to the structure of the world whatever that structure may be-the only constraint is the cardinality of the relevant domain. But to find out what that structure is presumably is something that requires careful empirical research rather than simply an exploration of the expressive resources of set theory.

Of course, this is just a particular set-theoretic formulation of the more general point that Newman raised. Interestingly, he did not rely on set theory to make his point, which is quite broad, and is not tied to any particular mathematical framework. This is the case despite his talk of "collections of things" (in the quotation above). 
After all, this can be understood in a variety of non-set-theoretic ways: for instance, as pluralities (various things) or as kinds (things that have certain traits in common)none of them depend on sets.

The limitation of the set-theoretic representation emerges from the fact that, whatever the structure of the world may turn out to be in the end, presumably it is not an abstract object. But it will be an object of this kind if it were identified with a set-theoretic structure. So, all things considered, it is better not to reify such representations and avoid taking the structure of the world as something that should be identified with a particular set.

There have been several attempts to respond to Newman's objection. In what follows, we will not review them. Rather, we list three significant ingredients that we will eventually use to articulate our own response.

\subsection{Three ingredients for a response to Newman's objection}

\subsubsection{Using the semantic approach}

Steven French and James Ladyman argue that the Newman objection does not arise in the framework of the semantic view of scientific theories: "the Newman problem is obviated if one does not think of structures and relations in first-order extensional terms" (French and Ladyman 2003, p.33). Although it is possible to show that, at least for a particular formulation of the semantic view, the Newman objections does arise (see Ainsworth 2009, pp.150-2), we think that it is an open issue whether all versions of structuralism within the semantic approach are affected by the Newman objection. This includes the partial structures approach (da Costa and French 2003), the structuralist meta-theory (Balzer, Moulines, and Sneed 1987), and structuralist empiricism (van Fraassen 2008). After all, Newman's formal result holds regardless of the mathematical framework that is used. However, we will argue below that one can avoid the Newman objection by making use of a specific form of the semantic conception, and the maneuver we offer can then be adopted by the defenders of all of these views.

Moreover, there is an important ingredient in French and Ladyman's response: if relations are not understood in first-order, extensional terms, there is more to whether they obtain or not than what is given by the extensions of any particular relation (the objects that are in the relation in question). Part of the problem with Newman's objection is that it assumes that there are virtually no constraints on what relations can be formed, as is the case with a purely extensional account of such relations. But such an account is clearly inadequate. For instance, the relations is taller than and is neither shorter than nor has the same height as arguably have the same extension, but they are not the same relations. So, additional, intensional constraints 
are needed to capture the relations under consideration, and the purely formal maneuver involved in Newman's objection is questioned. This is an important insight, and if properly implemented, as we will indicate below, it provides a significant ingredient to avoid the Newman problem.

\subsubsection{Empirical and non-empirical structures}

Ioannis Votsis proposes that we should distinguish between structures that are built up independently of experience (that is, a priori), and structures that are arrived at after empirical investigation (that is, a posteriori). This is a significant distinction in the context of structure building. It is part of one's understanding of scientific practice to make sense of the role played by empirical theories rather than just accommodating the formulation of abstract, purely mathematical structures. As Votsis argues:

Take the numbers 133 and 123. I can, restricting myself solely to arithmetic, perform various operations on these numbers. One such operation is addition. Similarly, if I had two collections of 133 and 123 physical objects respectively, I could count them one by one, and would reach the same result. Despite the similarities, there is an important difference between the two cases. The latter case is one in which the result is a property that is then ascribed to the physical world, in particular to the physical objects under consideration, and not merely an exercise of arithmetic. This claim is warranted by the employment of an empirical method to arrive at the given number (Votsis 2003, p.886).

The example illustrates the difference between structures that are formulated in purely mathematical terms and structures that are formulated in order to represent empirical information about the world. By performing purely formal operations on structures, the Newman objection emerges. But this changes once structures about physical systems, which are part of the world, are specified.

Some complain that the distinction between the way in which the relevant structures are arrived at, whether they depend on empirical investigation or not, cannot play the role that Votsis intends. As Peter Ainsworth points out:

it is not obvious why the fact that some structures have been arrived at a posteriori guarantees that these structures are more important than those structures that have been arrived at 'merely' a priori. Simply being arrived at via an a posteriori method does not seem to be sufficient to make a result important, especially if that result could have been arrived at a priori. After all, if the claim 'eggs is eggs' had been discovered to be true a posteriori, it would not thereby be more important than those identity claims that had been arrived at 'merely' a priori (Ainsworth 2009, p.167; italics in the original). 
The trouble with Ainsworth's response is that it fails to acknowledge the significance of the distinction between empirical and non-empirical systems. The fact that certain structures are obtained (or are determined) empirically highlights the nature of the information that shape and support them. As opposed to simple identity claims, which may hold quite independently of empirical considerations, it is precisely the contingency of the relevant empirical information that requires the use of empirical resources. This stresses the significance of the difference between the role played by structures that are characterized a priori and those that are arrived at a posteriori. Although the former can be formulated analytically and are based on logic and mathematics, the latter depend on how the world is: in order to settle empirical matters, structures of this sort play the decisive role.

A successful response to Newman's objection should explore the significance of this distinction. A purely formal result, despite its generality, may not be applicable to concrete cases which often involve additional constraints that turn the general result irrelevant or inapplicable. In the end, as will become clear below, Newman's formal result does not have an impact on actual formulations and reconstructions of scientific theories, which meet empirical conditions that are not captured by the formal result.

\subsubsection{Impure structuralism}

It should be acknowledged that purely formal features are often not enough to constrain significantly a philosophical view. As a philosophical conception, structuralism, in particular, is not pure. It relies crucially on traits that go beyond its formal underpinnings. These traits depend on how the relevant structures are interpreted and on what they are ultimately about.

Before proposing their own account, French and Saatsi note: "What saves the Ramseying structural realist is the fact that the theoretical content captured by her use of Ramseyfication goes well beyond the mere formal, logical structure of the unobservable world" (French and Saatsi 2006, p.551). They rightly emphasize that structuralism cannot be a purely formal view: "[...] we take it that in reality there has never been [...] a purely structural view of theories" (French and Saatsi 2006, p.557).

It is, in fact, a misinterpretation by the critics of structuralism to suppose that at issue is a purely structuralist conception. Something needs to be said about the empirical content and the intended applications of the theories under consideration. This trait makes the structuralist proposal advanced below at best partially structural—or impure.

The misinterpretation may have emerged from the historical context in which Newman's original critique was formulated: it was addressed to Russell's (1927) the- 
ory of perception, which was part of a very general epistemological account. Thus, the critique originated in a context that was significantly different from current debates about structuralism. Whereas Russell's conception was very broad and received a correspondingly broad critique, debates about contemporary structuralism crucially engage with particular formulations of scientific theories. Understanding the content of these theories is decisive to provide a defense of structuralism, but this means that there are concrete bits of information about them that cannot be abstracted away.

French and Saatsi emphasize this point:

It is not the case that any unobservable domain of suitable cardinality can be extensionally 'carved out' into isomorphic structure in a way that satisfies this constraint on the members of the domain [namely, that it is 'meaningful to speak of simultaneity and time-wise state-transitions' about them]. Yet, provided that this constraint is satisfied, it is meaningful to speak of representing the structure of the domain. [...] The theoretical variables for which the simultaneous values and their change are given by the structure are theoretically interpreted: they refer to physical properties and relations. It is not the case that all the theoretical content about these variables is encoded in the structure of their interrelations at each moment of simultaneity and over time-there is interpretational content about these properties that is captured linguistically (French and Saatsi 2006, pp.557-8; italics in the original).

Highlighted in this passage is the fact that, for any structural representation of an empirical domain, there is always reference to corresponding physical relations (and that content is captured linguistically as part of the proper formulation of the relevant structures). What this exactly amounts to will de developed below by invoking, and suitably interpreting, Carnap's founded relations.

\subsubsection{Toward a pragmatic account}

The three ingredients above-the use of the semantic approach (especially the rejection of a purely extensional understanding of relations), the distinction between empirical and non-empirical structures, and the understanding of structuralism as an impure conception-provide key features in resisting Newman's objection in the context of contemporary structuralism. As a purely formal result, there is no doubt that Newman's point goes through, but we challenge that it has the philosophical significance that its defenders have taken it to have.

Debates on structuralism (particularly with regard to ESR) involve the search for cases of structural continuity between apparently radically different theories. Given these cases, a variety of different formal tools can be invoked to reconstruct the theories in question or represent salient features of the epistemic context they are in- 
such as, for instance, Ramsey-sentence views or semantic approaches (partial structures, structuralist meta-theory, state-spaces, and so on). Thus, the semantic conception is particularly important in this context.

When the structure of an empirical theory is specified and interpreted in a given formal framework, Newman's problem does not emerge. After all, as part of that specification, it is explicitly stated what the domain of the theory consists of (particles, genes, markets, etc.), and it is no longer a trivial matter whether structures of the appropriate kind can be constructed. Thus, the role played by empirical structures is crucial.

It could be objected that this suggestion entails that rejection of structuralism, given the crucial role played by non-structural components: what theories are about, and the relations that are supposed to hold between them and the corresponding empirical domain. But this is not the case. After all, as noted above, structuralism needs to be formulated as an impure view, one that acknowledges the need for nonstructural components. What is highlighted here is a pragmatic maneuver, one that emphasizes the importance of paying attention to the application contexts in which structures used in the sciences are found. Once proper attention is played to this fact, the philosophical import of Newman's result is, at best, of limited significance.

Although epistemic structural realism is, in principle, affected by the Newman objection, it is less clear that the problem has an impact on ontic structural realism (OSR). After all, OSR is not an epistemological position, but rather a metaphysical view about what there is. The fundamental structures posited by OSR are what they are independently of what one takes them to be. Whether or not structural descriptions only constrain the cardinality of what can be known about the world is not something that alters the view. OSR does not involve any particular epistemological account: it does not involve claims about the structure of one's knowledge of the world or, more specifically, about the structure of the formal representations of one's knowledge of the unobservable parts of the world. Thus, even if the Newman objection went through, it would be ultimately irrelevant for OSR. (One can, of course, press the issue of what is the suitable epistemology for OSR. But this is a separate concern, which, of course, needs to be addressed by any full defense of the view.)

\section{A Pragmatic Approach to the Newman Objection}

With these considerations in place, we can now proceed to develop our own account of what can be called "pragmatic structuralism". 


\subsection{A pragmatic proposal}

As is well known, semantic approaches in the philosophy of science typically make use of set-theoretic predicates, relying on Suppes' (1957) original proposal. We can characterize a set-theoretic predicate $K$ as a predicate that specifies:

- the type of a structure $\left\langle D_{1}, \ldots, D_{k}, R_{1}, \ldots, R_{n}\right\rangle$, where $k$ is the number of base sets, and $n$ the number of relations;

- the typification of the relations $R_{1}, \ldots, R_{n}$;

- the axioms that the relations $R_{1}, \ldots, R_{n}$ need to satisfy.

The structure $\left\langle D_{1}, \ldots, D_{k}, R_{1}, \ldots, R_{n}\right\rangle$ will eventually satisfy the set-theoretic predicate $K$. This methodological tool allows one to represent the structure of scientific theories (for instance, within ESR).

One standard example of such a representation in the formal framework of the structuralist meta-theory can be presented in the following way. Consider the potential model of Classical Collision Mechanics (see Balzer, Moulines, and Sneed 1987). The model, $M_{p}(C C M)$, is formulated as $\langle P, T, \mathbb{R}, v, m\rangle$, such that:

1. $P$ is a finite, non-empty set;

2. $T$ contains exactly two elements;

3. $v: P \times T \rightarrow \mathbb{R}^{3}$;

4. $m: P \rightarrow \mathbb{R}^{+}$.

The basic intended interpretation is one in which $P$ is a set of discrete bodies that can be called 'particles'. $T$ is a set of two instants, one time instant before the collision and one after it. Moreover, $v$ is the velocity function, assigning to each particle $p$ and point in time its velocity as an element of $\mathbb{R}^{3}$. Velocity is then a time-dependent vectorial function and its range is identified with triples of real numbers. It assigns, to each particle and at each instant in time, a three-component vector (one component for each direction in space). Finally, $m$ is the mass function, assigning to each particle its mass.

As opposed to what one may be led to believe in light of Newman's objection, the information provided by the formulation of classical collision mechanics in terms of a set-theoretic predicate is far from trivial. The potential model above clearly specifies the elements of the domain: they are particles. And since a structure of the relevant kind-about the empirical objects in question-may not be available, the triviality charge can be resisted. This is achieved by invoking a particular pragmatic stance: it consists in specifying the target empirical system, and to determine concretely the 
domain of objects and relations among them. This is required to ground the structural formulation of the theory and to avoid turning a mathematized scientific theory into just a piece of mathematics. Clearly, this proposal bears relations to van Fraassen's (1997) response to Putnam's model-theoretic argument, but as will become clear below, it is also importantly different from it.

In purely formal terms, Newman's objection challenges those who claim that "all one's knowledge of the world is structural". But the proper understanding of the relevant scientific theories requires attention to their pragmatic counterpart. Thus, consideration of the particular applications of these theories is central. Such consideration is crucially highlighted by the semantic view, given that the structures that are used in the formulation of scientific theories need to be interpreted and connected to suitable empirical domains. In fact, this is precisely one of the central roles of simple pragmatic structures in the partial structures approach (da Costa and French 2003) and potential models in the structuralist meta-theory (Balzer, Moulines, and Sneed 1987). Given the need to specify explicitly what the relevant theories are about (as representations of the phenomena under consideration), that is, given the role of empirical considerations in the interpretation of structures, the information encoded by the relevant structures is ultimately not trivial.

\subsection{Carnap's founded relations}

That empirical considerations are central to the interpretation of structures in science is an empiricist sentiment that has been articulated very clearly by Carnap. In $\S 154$ of the Aufbau (Carnap 1928), he introduces founded relations. As he notes: "We wish to call relation extensions which correspond to experienceable, 'natural' relations founded relation extensions" (Carnap 1928, p.236). It is crucial, for this conception, that founded relations be erlebbar, that is, that they be experienceable-they can be experienced. They are natural in the sense that they are not made up, gerrymandered, or manipulated in artificial ways.

The relations, understood in intensional terms, which are specified by the relevant set-theoretic predicates that are used in the formulation of a given scientific theory, are experienceable and "natural" in Carnap's sense. In light of this, when interpreting the relevant structures, the relations that are specified and that are invoked in the description of the domain in question are those corresponding to experienceable relations. Interpreted in this way, the resulting structures are no longer purely mathematical, but correspond to and offer descriptions of experienceable relations.

Carnap considers founded relations not to be empirical items, but rather logical ones. As he notes: "It is perhaps permissible, because of [its] generality, to envisage the concept of foundedness as a concept of logic and to introduce it, since it is undefinable, as a basic concept of logic" (Carnap 1928, p.237). This is surprising since 
the generality of foundedness is not enough to make it a logical notion. Object is a similarly general concept, but arguably it is not a logical one (some logical systems, such as propositional logics, have no room even to express such a notion). More importantly, it is not clear how founded relations, if taken to be logical, could be used to provide any kind of constraint on admissible interpretations of structures. After all, logical notions, at least as they are usually understood, hold universally, and thus will fail to rule out any empirical situation. This would make them unfit to block any triviality concerns. (For additional discussion of this point, see Bueno 2017.) To avoid these difficulties, as opposed to Carnap, we do not interpret founded relations as a logical notion, but rather take them to be empirical. We do not take founded relations to be in the realm of Carnapian rational reconstructions, but rather in the realm of experience. The role played by pragmatics in this context is something that Carnap certainly approves.

With these considerations in place, it is now possible to provide an answer to the Newman objection by restricting the interpretation of the relevant structures to intentional relations of an experienceable sort. Some of these relations can be experienced directly (such as those that are directly observable); others can only be experienced indirectly (via instrumental access). But none of them are abstract. Relations of this kind, which are neither causally active nor spatiotemporally located, cannot be experienced. But provided that the relations that are interpreted are experienceable, triviality concerns can be addressed. In the end, it is the combination of empiricism (with the emphasis on empirical structures), the use of pragmatic traits in an impure form of structuralism (with the distinctive role played by the interpretation of the relevant structures) together with the semantic approach (and the intensional understanding of the relations in question) that allows one to resist Newman's objection.

\subsection{Structural scrutability}

David Chalmers (2012) proposes a way out of the Newman problem that shares some features with the pragmatic approach we favor. He considers Carnap's original conception of pure structuralism as being insufficient to the task at hand, and argues that:

[...] this leaves open the possibility of weak structuralism, on which the basic vocabulary may include a limited number of expressions for relations (such as phenomenal similarity) plus logical expressions.

Both theses [regarding the characterizations of, respectively, pure structuralism and weak structuralism] have analogs in the domain of scrutability. The analog of pure structuralism is Logical Scrutability: the thesis that all truths are scrutable from truths using logical vocabulary alone. The analog 
of weak structuralism is Structural Scrutability: roughly, the thesis that all truths are scrutable from truths using logical vocabulary plus structural expressions, where (to a first approximation) a structural expression is one that expresses a basic relation. Logical Scrutability is undermined by Newman's problem, but Structural Scrutability remains on the table (Chalmers 2012, p.409).

An analogy is suggested here between weak structuralism and structural scrutability. Whereas our pragmatic structuralism meshes well with weak structuralism, it is clearly distinct from structural scrutability, since pragmatic structuralism does not rely on any notion of truth, nor does it aim at connecting any truth-related notion with a structuralist stance. This is an important difference. The notion of truth, or any similar notion, such as truthlikeness, is part of a realist view, and the proposal we advance aims to leave open possible anti-realist proposals, such as empiricist ones. The focus, it should be clear, is on structural descriptions, implemented in the pragmatic way just outlined. And as we will argue in the next section, the approach we offer, depending on how it is interpreted, provides a family of answers to the Newman problem, answers that are compatible with a variety of different approaches to the realism/anti-realism debate.

Chalmers (2012, p.411) also argues that by explicitly specifying the physical system under consideration, it is possible to save the resulting construction from Newman's problem. This maneuver is similar to the one we favor, since, as argued above, one needs to specify, in each case, the relevant physical system under consideration. But, in contrast to Chalmers' proposal, the success of such specification does not rely on any truth-related notion.

\section{Structuralism, Empiricism, and Realism}

By making the pragmatic maneuver we recommend, have we given up on pure structuralism when experienceable relations are invoked? The answer is affirmative, since pragmatic considerations are not considered to be part of a pure structuralist framework. But without such a pragmatic component, structuralism makes very little sense, since one cannot specify what the structures that are introduced in one's theories are about. The intelligibility of structuralism ultimately demands such component.

Is realism rejected by focusing on experienceable relations? Throughout this paper, we have emphasized the empiricist requirement on such relations, but we have deliberately understood such relations very broadly. As noted above, some such relations are directly experienceable, others are only indirectly so. Thus, the proposal here actually provides a broad framework that delivers a family of responses to Newman's objection depending on how experienceable relations are understood. Three 
possible interpretations can be offered:

1. Constructive empiricist reading: The experienceable relations-those to which one should assign an epistemic role-are restricted only to directly observable relations (van Fraassen 1980 and 2008). Given that, for the constructive empiricist, what is observable is what can be seen with one's naked eyes, experienceable relations are understood very narrowly. But it is no trivial matter to provide structures that generate directly observable information about a given domain. The Newman objection can then be resisted.

2. Broadly empiricist reading: The experienceable relations include directly observable relations and indirectly observable relations-as long as one knows (or, at least, has good reasons to believe) that the relevant instruments satisfy epistemic conditions akin to observation (Bueno 2017). To obtain structures that yield information about what is directly observable or observable through suitable scientific instruments is no trivial task. There is no guarantee that any such structures can be determined at all, and in this way, the triviality concern is also addressed.

3. Realist reading: The experienceable relations include directly observable relations and indirectly detectable relations, that is, one can detect them with instruments that need not be akin to observations (Chakravartty 2007 and French 2014). These are clearly realist views, which support commitment to structures that go well beyond anything that the previous two options would maintain. But these views can still resist the Newman problem, given that it is no trivial matter whether there are structures that offer information about relations that are detectable in this sense.

Thus, depending on how one interprets the experienceable relations, realist and antirealist views can be accommodated within the framework we propose, and correspondingly different responses to Newman's problem emerge. What we have here is, thus, a family of possible responses, which vary in accordance with the interpretation of scientific activity that is adopted.

\section{Conclusion}

Newman's objection holds if restricted to pure, abstract structural claims. But understood in this way, the objection has no connection to structuralism in the philosophy of science, where concrete applications to the physical world are crucially involved. When invoking a set-theoretic predicate formulation of a scientific theory, one needs to state explicitly the domain of objects the theory is about. In doing so, one is forced 
to leave behind the territory of abstract structures and provide empirical interpretations of them. One has then to emphasize the crucial role of experienceable relations, and advance a suitable setting in which these relations can be interpreted.

This leads to a framework able to accommodate a variety of philosophical views, such as constructive empiricism, a broad form of empiricism, and realism, all of which are able to respond to the Newman objection. In this way, it is possible to acknowledge the place of this objection as a purely formal result, while noting that its philosophical impact is, in the end, very limited. The objection may present a problem for a very abstract form of structuralism, perhaps such as the one that was entertained by Russell 1927, but it does not affect structuralism about scientific theories more generally. Empirical relations are crucial for the latter, thus providing resources to prevent the intended trivialization from getting off the ground.

\section{References}

Ainsworth, P. 2009. Newman's Objection. British Journal for the Philosophy of Science 60: $135-71$.

Arenhart, J.; Bueno, O. 2015. Structural Realism and the Nature of Structure. European Journal of Philosophy of Science 5: 111-39.

Balzer, W.; Moulines, C. U.; Sneed, J. 1987. An Architectonic for Science. Dordrecht: Reidel.

Bueno, O. 1999. What is Structural Empiricism? Scientific Change in an Empiricist Setting. Erkenntnis 50: 59-85.

Bueno, O. 2017. Overcoming Newman's Objection. In: M. Massimi; J.-W. Romeijn; G. Schurz (eds.) EPSA 15: Selected Papers, pp.3-12. Dordrecht: Springer.

Chakravartty, A. 2007. A Metaphysics for Scientific Realism: Knowing the Unobservable. Cambridge: Cambridge University Press.

Carnap, R. 1928[1967]. The Logical Structure of the World. (English translation by Rolf A. George.) Berkeley: University of California Press.

Chalmers, D. 2012. Constructing the World. Oxford: Oxford University Press.

da Costa, N. C. A.; French, S. 2003. Science and Partial Truth: A Unitary Approach to Models and Scientific Reasoning. New York: Oxford University Press.

Demopoulos, W.; Friedman, M. 1985. Bertrand Russell's The Analysis of Matter: Its Historical Context and Contemporary Interest. Philosophy of Science 52: 621-39.

French, S. 2014. The Structure of the World: Metaphysics and Representation. Oxford: Oxford University Press.

French, S.; Ladyman, J. 2003. Remodelling Structural Realism: Quantum Physics and the Metaphysics of Structure. Synthese 136: 31-56.

French, S.; Saatsi, J. 2006. Realism about Structure: The Semantic View and Nonlinguistic Representations. Philosophy of Science 73: 548-59.

Frigg, R.; Votsis, I. 2011. Everything you Always Wanted to Know about Structural Realism but Were Afraid to Ask. European Journal for Philosophy of Science 1(2): 227-76.

Ladyman, J. 1998. What is Structural Realism? Studies in History and Philosophy of Science 29: 409-24. 
Newman, M. H. A. 1928. Mr. Russell's 'Causal Theory of Perception'. Mind 37: 137-48.

Russell, B. 1927. The Analysis of Matter. London: Kegan and Paul.

Suppes. P. 1957[1999]. Introduction to Logic. New York: Dover.

van Fraassen, B. C. 1980. The Scientific Image. Oxford: Clarendon Press.

— 1997 Putnam's Paradox: Metaphysical Realism Revamped and Evaded. Philosophical Perspectives 11: 17-42.

2008. Scientific Representation: Paradoxes of Perspective. Oxford: Clarendon Press.

Votsis, I. 2003. Is Structure Not Enough? Philosophy of Science 70: 879-90.

\section{Acknowledgments}

Our thanks go to an anonymous reviewer for this journal for helpful comments on an earlier version of this work. 